\section{Brigatinib bei ALK-positivem NSCLC}

\author{
Die meisten mit Crizotinib behandelten Patienten mit ALK-positivem NSCLC \\ erleiden in absehbarer Zeit ein Rezidiv. In dieser Studie wurden zwei Dosen \\ des Zweitgenerations-ALK-Inhibitors Brigatinib bei solchen Patienten geprüft.
}

$\mathrm{R}_{x \rightarrow 1}$ und $5 \%$ der Patienten mit nichtkleinzelligem Lungenkarzinom (NSCLC) haben ein ALK-Rearrangement und profitieren von Crizotinib durch hohe Remissionsraten (> 60\%) und ein verlängertes progressionsfreies Überleben (PFS). Praktisch immer kommt es zu einer Resistenz; neben Ceritinib und Alectinib ist Brigatinib - alle ALK-Inhibitoren der der zweiten Generation - wegen Überwindung der meisten Resistenzmutationen besonders erfolgversprechend. In einer randomisierten Phase-II-Studie erhielten 222 Patienten mit ALK-positivem NSCLC und Progress unter Crizotinib 90 oder 180 mg/d Brigatinib, wobei im Arm mit 180-mg-Dosierung während der ersten Woche $90 \mathrm{mg}$ gegeben wurden. $69 \%$ der Patienten hatten zu Beginn Hirnmetastasen, auf die primäre Crizotinib-Therapie hatten etwa zwei Drittel angesprochen.

Unter $90 \mathrm{mg}$ Brigatinib betrug die von den Untersuchern bestimmte Remissionsrate (überwiegend partiell) $45 \%$, unter $180 \mathrm{mg} 54 \%$. Die Krankheitskontrollrate lag bei 82 bzw. $86 \%$. Das PFS wurde mit 9,2 bzw. 12,9 Monaten bestimmt und die PFS-Wahrscheinlichkeit nach 6 Monaten betrug 63 bzw. 78\%. Nach einem Jahr lag die Überlebenswahrscheinlichkeit bei $71 \%$ im 90-mg-
Arm und bei $80 \%$ im 180-mg-Arm. Von den Patienten mit aktiven Hirnmetastasen erzielten $42 \%$ unter $90 \mathrm{mg}$ Brigatinib und $73 \%$ unter $180 \mathrm{mg}$ Brigatinib eine ZNS-Remission, das mediane intrakranielle PFS lag bei 15,6 bzw. 12,8 Monaten.

Fazit: Brigatinib hat bei Patienten mit ALK-positivem NSCLC selbst nach Versagen einer Crizotinib-Therapie noch eine hohe und klinisch relevante Wirksamkeit. Neben günstigen Remissionsraten und einem vergleichsweise langen PFS konnte eine Wirksamkeit auch bei Hirnmetastasen nachgewiesen werden. $180 \mathrm{mg}$ Brigatinib erwiesen sich als effektiver als die 90-mg-Dosis - bei akzeptabler Verträglichkeit. Brigitte Schalhorn

Kim DW et al. Brigatinib in patients with crizotinib refractory anaplastic lymphoma kinasepositive non-small-cell lung cancer: a randomized, multicenter phase II trial. J Clin Oncol. 2017;35(22):2490-8

\title{
Ceritinib beim ROS1-positiven NSCLC
}

Nur etwa 1-2\% aller nichtkleinzelligen Lungenkarzinome (NSCLC) weisen ein ROS1-Rearrangement auf. Wegen der großen strukturellen Ähnlichkeiten der Kinasedomänen von ROS1 und ALK profitieren die Patienten ähnlich gut von ALK-Inhibitoren wie Betroffene mit ALK-positiven Tumoren. Welche Remissionsraten lassen sich mit dem ALK-Inhibitor Ceritinib erreichen?

n einer multizentrischen koreanischen Phase-II-Studie wurden 404 Patienten gescreent, von denen 34 FISH-positiv für eine ROS1-Translokation waren. Sie erhielten $750 \mathrm{mg}$ des ZweitgenerationsALK-Inhibitors Ceritinib pro Tag oral. Primärer Endpunkt war die Remissionsrate, und sie betrug nach median $14 \mathrm{Mo-}$ naten in der Intention-to-Treat(ITT)-
Analyse $62 \%$. 1 Patient zeigte ein komplettes Ansprechen, 19 ein partielles (Tab. 1); die Remissionsdauer lag bei 21 Monaten und die Krankheitskontrollrate bei $81 \%$. Bei drei Viertel der Patienten konnte ein Rückgang der Tumorlast erzielt werden. Das mediane progressionsfreie Überleben der gesamten Patientengruppe betrug 9,3 Monate und bei den

Tab. 1: Aktivität von Ceritinib (Auswahl)*
\begin{tabular}{l|l|l} 
Bestes Ansprechen & Alle Patienten (n=32), $\mathbf{n}(\%)$ & Crizotinib-naive Patienten (n=30), $\mathbf{n}(\%)$ \\
\hline CR & $1(3 \%)$ & $1(3 \%)$ \\
\hline PR & $19(59 \%)$ & $19(63 \%)$ \\
\hline SD & $6(19 \%)$ & $6(20 \%)$ \\
\hline PD & $2(6 \%)$ & $2(7 \%)$
\end{tabular}

$C R=$ komplettes Ansprechen; $P R=$ partielles Ansprechen; $S D=$ stabile Erkrankung; $P D=$ progressive Erkrankung; * durch unabhängiges Komitee erhoben zuvor noch nicht mit einem ALK-Inhibitor (Crizotinib) behandelten Patienten 21 Monate. Die Gesamtüberlebensrate lag nach 6 Monaten bei $84 \%$ und nach 12 Monaten bei $56 \%$. Das mediane Gesamtüberleben betrug 24 Monate. Von den wenigen Patienten mit Hirnmetastasen $(\mathrm{n}=8)$ profitierten 5 zumindest von einer Krankheitskontrolle.

Eine Dosisreduktion aufgrund von Nebenwirkungen wurde bei $68 \%$ und eine Therapieunterbrechung bei $72 \%$ der Patienten erforderlich, es fand sich kein Fall einer Pneumonitis oder einer QTcVerlängerung.

Fazit: Ceritinib erwies sich bei mehrfach vorbehandelten Patienten mit einem ROS1-positiven NSCLC als sehr effektiv. Es führte zu hohen Remissionsraten, zu oft länger anhaltenden Remissionen und $\mathrm{zu}$ einem langen progressionsfreien Überleben. Selbst die wenigen Patienten mit Hirnmetastasen konnten zum Teil von diesem ALK-Inhibitor der zweiten Generation profitieren.

Brigitte Schalhorn

Lim SM et al. Open-label, multicenter, phase II study of ceritinib in patients with non-small-cell lung cancer haboring PROS1 rearrangement. J Clin Oncol. 2017;35(23):2613-8. 